\title{
Long-term Survival and Propensity Score Matched Outcomes of Bilateral vs. Unilateral Diaphragm Interventions in Cytoreductive Surgery plus Intra-peritoneal Chemotherapy
}

\author{
BHAVNEET SINGH ${ }^{1,2}$, GURKIRAT SINGH ${ }^{1}$, MOHAMMAD POURGHOLAMI $^{2}$, \\ NAYEF ALZAHRANI ${ }^{2,3}$ and DAVID L. MORRIS ${ }^{2}$ \\ ${ }^{1}$ MBBS, University of Western Sydney, Sydney, Australia; \\ ${ }^{2}$ UNSW Department of Surgery, St. George Hospital, Sydney, Australia; \\ ${ }^{3}$ Al Imam Mohammad Ibn Saud Islamic University, Riyadh, Saudi Arabia
}

\begin{abstract}
Background/Aim: To assess the impact of shortand long-term outcomes of bilateral vs. unilateral diaphragm interventions in cyto-reductive surgery (CRS) and intraperitoneal chemotherapy (IPC). Patients and Methods: A total of 652 CRS/IPC procedures, between 1996 and 2018, required diaphragm interventions. Among these, 388 underwent bilateral intervention. Preoperative heterogeneity was assessed in 6 parameters and addressed with propensity score matching. The association of each respective analysis was assessed with 11 outcomes. Overall survival was assessed based on histology. Results: CRS/IPC requiring bilateral diaphragmatic interventions illustrated significantly increased operative hours (9.6 vs. 8.6 hours, $p<0.001$ ). Postoperatively, there was significantly increased red blood cell (RBC) transfusion (6.37 units vs. 4.47 units, $p=0.007$ ) and grade III and IV complications $(57.3 \%$ vs. $40.6 \%, p=0.004)$. No difference was noted in ICU stay, total length of stay, hospital death and return to OT. In terms of respiratory complications, an increased incidence of pneumothorax (16.5\% vs. 6.2\%, $p<0.001)$ was noted whilst pleural effusions and pneumonia occurrences were non-significant. Overall survival, revealed bilateral interventions in low-grade appendiceal mucinous neoplasm conferred an increased relative risk ( $p=0.037$, $R R=2.230,95 \% C I=1.052-4.730$ ). They did not have an effect on $\mathrm{OS}$ in colorectal cancer and mesothelioma. Conclusion: Despite the increase in short-term morbidity, bilateral diaphragm interventions resulted in similar long-term survival to unilateral interventions.
\end{abstract}

Correspondence to: Bhavneet Singh, 30 Gray Street, Kogarah, NSW, 2217, Sydney, Australia. Tel: +61405832619, e-mail: Bavsingh8@gmail.com

Key Words: Cyto-reductive, intra-peritoneal chemotherapy, diaphragm.
Selected patients with peritoneal carcinomatosis are treated with cyto-reductive surgery and intra-peritoneal chemotherapy (CRS/IPC) to increase longevity. Its efficacy has been proven in low-grade appendiceal mucinous neoplasms (LAMNs) (1), high-grade appendiceal mucinous neoplasms (HAMNs) (1), peritoneal mesothelioma (2) and ovarian cancer (3), colorectal cancer $(4,5)$ and other rare etiologies.

The goal of cyto-reduction is to remove all macroscopic disease. It is often complex and involves multiple abdominal regions. The administration of intra-peritoneal heated chemotherapy targets residual microscopic disease. Subsequently, the combination of a technically challenging procedure with the cytotoxicity of chemotherapy (6) results in a high rate of perioperative mortality $(0.37-4.1 \%)$ and morbidity $(10-33 \%)(7,8)$.

Diaphragmatic intervention is needed in $50 \%$ of CRS/IPC procedures. Retrospective single institution studies have demonstrated morbidity attributable to diaphragmatic interventions. This includes longer surgical times, resections and increased complication rates $(9,10)$.

The aim of this study was to evaluate whether diaphragmatic resection confers a higher short-term or longterm mortality in CRS/IPC. Firstly, perioperative morbidity was assessed. Secondly, by assessing overall survival it was established whether the short-term morbidity was acceptable.

\section{Patients and Methods}

This is a retrospective cohort study conducted in a single highvolume center, St. George Hospital, Sydney, from a prospectively maintained database. Because this was regarded as service development by the local research and development committee, formal ethical approval was not required. A database dated from September 1996 to March 2018, of all CRS/IPC for peritoneal-based malignancies, was searched. A multi-disciplinary team determined suitability for CRS/IPC. Suitability was assessed based on disease histology, extent, ability to achieve complete cytoreduction, 
Table I. Preoperative characteristics; bilateral vs. unilateral.

\begin{tabular}{|c|c|c|c|}
\hline Co-variate & $\begin{array}{c}\text { Bilateral } \\
388\end{array}$ & $\begin{array}{c}\text { Unilateral } \\
262\end{array}$ & $p$-Value \\
\hline ASA & & & 0.28 \\
\hline 1 & 17 & 10 & \\
\hline 2 & 95 & 69 & \\
\hline 3 & 198 & 143 & \\
\hline 4 & 38 & 15 & \\
\hline Gender & & & 0.83 \\
\hline M & 170 & 117 & \\
\hline $\mathrm{F}$ & 218 & 145 & \\
\hline Diagnosis & & & $<0.001 *$ \\
\hline Colorectal & 36 & 87 & $<0.001 *$ \\
\hline LAMNs & 125 & 70 & 0.13 \\
\hline Mesothelioma & 45 & 19 & 0.07 \\
\hline HAMNs & 146 & 46 & $<0.001 *$ \\
\hline Others & 12 & 21 & 0.01 \\
\hline Ovarian & 24 & 19 & 0.59 \\
\hline PSS & & & $<0.001 *$ \\
\hline 0 & 157 & 49 & $<0.001 *$ \\
\hline 1 & 98 & 96 & $<0.001 *$ \\
\hline 2 & 114 & 94 & 0.07 \\
\hline 3 & 15 & 18 & 0.08 \\
\hline \multicolumn{4}{|l|}{ Age } \\
\hline Mean & 53.05 & 53.68 & 0.556 \\
\hline SD & 13.56 & 13.09 & \\
\hline \multicolumn{4}{|l|}{ PCI } \\
\hline Mean & 27.63 & 15.20 & $<0.001 *$ \\
\hline SD & 8.86 & 8.84 & \\
\hline
\end{tabular}

ASA: American Society of Anesthesiologists physical status classification; PSS; prior surgical score; PCI: peritoneal cancer index; HAMNs: high-grade appendiceal mucinous neoplasms; LAMNs: lowgrade appendiceal mucinous neoplasms; SD: standard deviation.

performance status and comorbidities. Subsequent CRS/IPC was preformed utilizing the Sugarbaker technique (11).

The database consisted of a total of 1,230 patients. A total of 635 patients was identified, with the only inclusion criterion being diaphragmatic intervention. The diaphragm intervention group was further subdivided into interventions that were bilateral or unilateral.

Demographic data, intraoperative details and postoperative complications were extracted from the database. The extent of peritoneal disease was documented via the peritoneal carcinomatosis index (PCI) described by Jacquet and Sugarbaker (12). Completeness of cytoreduction (CC) was recorded in a similar manner (12). Post-operative complications were graded according to the Clavien-Dindo classification (13). Prior surgical score was documented as described by Jacquet et al. (12).

Statistical analysis was preformed using R 3.1.0 and SPSS version 23 (IBM corporation, New York, USA). Univariate analysis was utilized to identify heterogeneity with regards to 6 preoperative variables; including ASA, sex, diagnosis, PSS, age and PCI. Any significant heterogeneity was adjusted for with propensity score analysis utilizing the matchit package on R. Independent samples T-test was utilized for continuous variables and expressed as mean and SD. Categorical variables were assessed using the chi-square
Table II. Bilateral diaphragm versus unilateral intervention.

\begin{tabular}{lcccc}
\hline Co-variable & Unit & Bilateral & Unilateral & $p$-Value \\
\hline Non-PPM & Count & 388 & 262 & \\
PPM & Count & 165 & 165 & \\
Operative hours & Mean & 9.60 & 8.60 & $<0.001^{*}$ \\
& SD & 2.490 & 2.716 & \\
RBC & Mean & 6.37 & 4.48 & $0.007^{*}$ \\
& SD & 7.012 & 5.432 & \\
CC Score & Count & & & 0.742 \\
& 0.1 & 158 & 158 & \\
Pneumonia & Count & 15 & 13 & 0.731 \\
Pleural effusion & Count & 53 & 46 & 0.463 \\
Pneumothorax & Count & 27 & 10 & $0.004 *$ \\
Return to OT & Count & 33 & 30 & 0.734 \\
Hospital Death & Count & 2 & 5 & 0.280 \\
Morbidity grade & Count & & & $0.004 *$ \\
& 3.4 & 86 & 58 & \\
ICU days & Mean & 3.19 & 2.73 & 0.265 \\
& SD & 4.307 & 2.987 & \\
Length of stay & Mean & 21.01 & 25.12 & 0.062 \\
& SD & 18.75 & 21.051 & \\
\hline
\end{tabular}

RBC: Red blood cells; OT: operating theatre; SD: standard deviation; CC Score: completeness of cytoreduction; PPM: propensity matched.

test and expressed as frequencies and proportions. Where the chisquare analysis was violated, fishers exact test was utilized.

Overall survival (OS) was estimated using the non-matched data sets from the date of CRS/IPC to the date of death or last followup. The log-rank test compared survival between groups using the Kaplan-Meier method analysis. Cox regression was performed and accounted for the following co-variates; ASA, PSS, PCI and CC score. Assumptions were tested with covariates as a function of time and graphically via $\log$ minus $\log$ plots and scatter plots.

\section{Results}

Patient characteristics. A total of 1,230 patients underwent CRS/IPC through the study period. Among these, 652 (53.0\%) patients underwent concomitant diaphragmatic procedures. The mean age of patients was $56.3+12.2$ (range $=14-85$ years). There were $545(44.3 \%)$ male individuals. The mean PCI was $16.82+11.64$ (range=0-39). The histology of the primary tumor was colorectal 364 (29.6\%), HAMNs 319 (25.9\%), LAMNs 296 $(24.1 \%)$, mesothelioma $(8.3 \%)$, other $(7.6 \%)$, ovarian $(4.5 \%)$.

Table I provides a thorough summary of the pre-operative diversity between the bilateral and unilateral cohorts. Precisely, 388 (59.5\%) patients underwent bilateral diaphragm intervention and $262(40.2 \%)$ patients underwent unilateral procedures. Univariate analysis revealed significant differences in diagnosis of cancer type, prior surgical score (PSS) and PCI. Patients with LAMNs (37.6\% vs. $17.6 \%$, $p<0.001)$ were more likely to undergo bilateral intervention, 


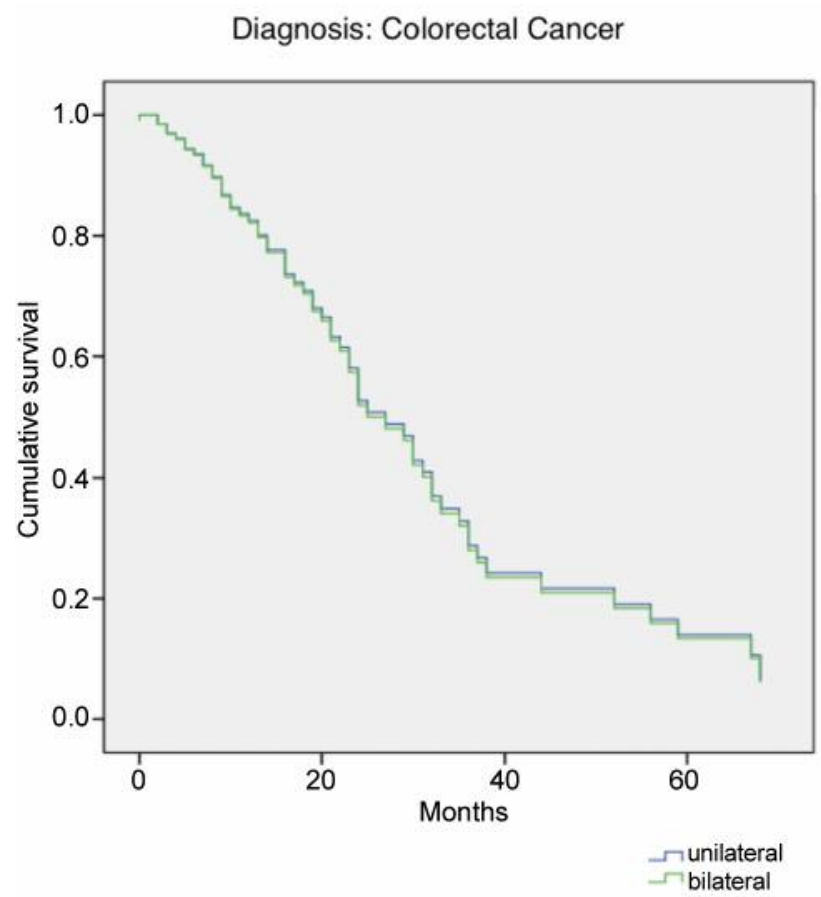

Figure 1. Overall survival of colorectal cancer patients undergoing bilateral diaphragm interventions versus unilateral diaphragm interventions.

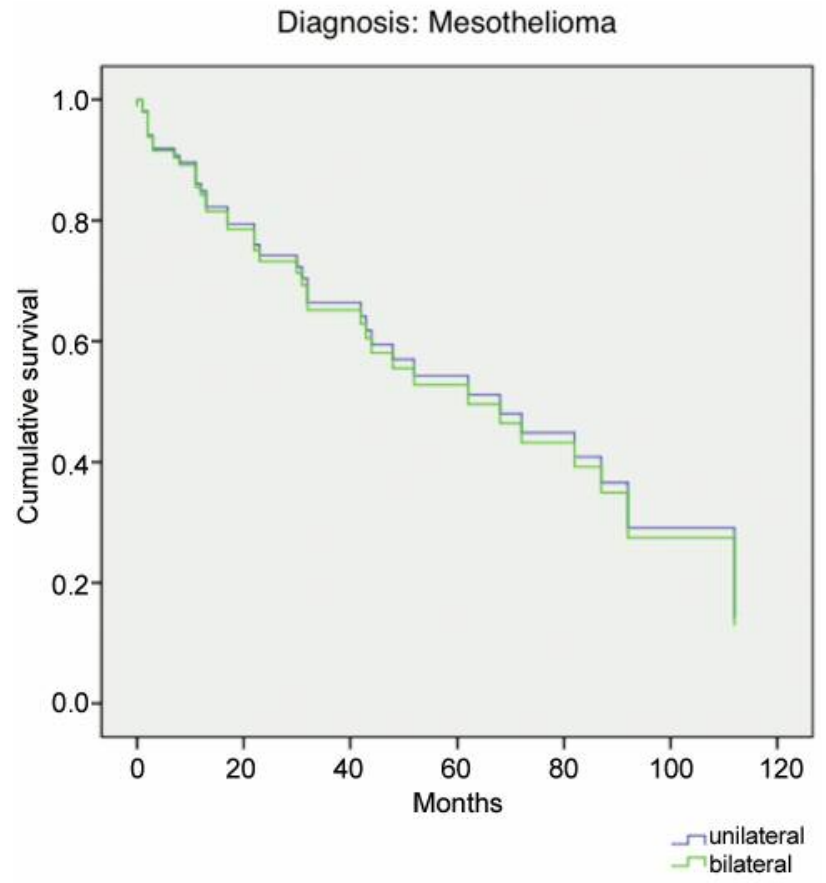

Figure 2. Overall survival of mesothelioma patients undergoing bilateral diaphragm interventions versus unilateral diaphragm interventions.
Diagnosis: Low Grade Appendiceal Neoplasms

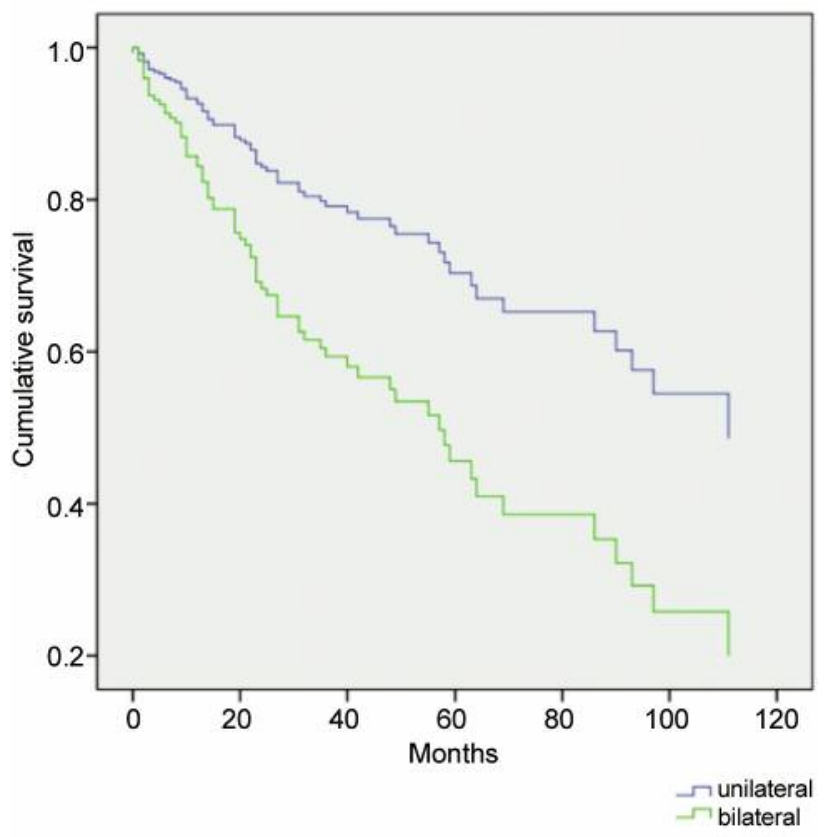

Figure 3. Overall survival of patients with LAMNs undergoing bilateral diaphragm interventions versus unilateral diaphragm interventions.

whereas colorectal cancer patients $(9.3 \%$ vs. $33.2 \%, p<0.001)$ were unlikely. Significantly increased numbers of patients with PSS 0 (40.9\% vs. 19.1\%, $p<0.001)$ underwent bilateral intervention, whereas patients with PSS 1 (25.5\% vs. 37.4\%, $p<0.001)$ was less likely to. Unsurprisingly, the mean PCI was considerably higher in the bilateral diaphragmatic group (27.6 vs. 15.2, $p<0.001$ ). The data were propensity matched for diagnosis, PSS and PCI and yielded 165 matched pairs.

Mortality and morbidity data. Bilateral diaphragmatic interventions in CRS/IPC were compared to unilateral intervention. Table II provides a thorough summary of our propensity matched univariate analysis. Intraoperative results illustrated significantly increased operative hours (9.6 vs. 8.6, $p<0.001)$ but no difference in CC score. Postoperatively, there was a significant increase in the incidence of red blood cell $(\mathrm{RBC})$ transfusion in bilateral $v s$. unilateral intervention (6.37 units vs. 4.47 units, $p=0.007$ ) as well as in grade III and IV complications ( $57.3 \%$ vs. $40.6 \%, p=0.004)$. No difference was noted in ICU stay, total length of stay, hospital death and return to operating theatre (OT). In terms of respiratory specific complications, a significantly increased incidence of pneumothorax $(16.5 \%$ vs. 6.2\%, $p<0.001$ ) was noted, whilst pleural effusions and pneumonia occurrences were not significant. 
Overall survival results. OS was analyzed on non-matched data and according tumor subtypes. Event rates only allowed comparison in three tumor types: colorectal, mesothelioma and LAMNs.

With regards to diaphragm interventions in colorectal cancer, 36 patients underwent bilateral and 87 unilateral intervention. At the time of the study 67 patients had died from disease. OS was unaffected in diaphragm interventions on Kaplan-Meier analysis (24 vs. 30 months median survival, $p=0.467)$. Similarly, on cox regression, diaphragm resection insignificantly affected long-term survival in colorectal cancer $(p=0.945, \mathrm{RR}=1.022,95 \% \mathrm{CI}=0.554-1.884$, Figure 1$)$.

With regards to diaphragm interventions in mesothelioma, 45 patients underwent bilateral and 19 unilateral intervention. At the time of the study 42 patients had died from disease. OS was unaffected in diaphragm interventions on Kaplan-Meier (32 vs. 68 months median survival, $p=0.685$ ). Similarly, on cox regression, diaphragm resection insignificantly affected long-term survival in mesothelioma $(p=0.926, \mathrm{RR}=1.046,95 \% \mathrm{CI}=0.403-2.713$, Figure 2$)$.

Regarding diaphragm interventions in LAMNs, 146 patients underwent bilateral and 46 unilateral interventions. At the time of the study 79 patients had died from disease. OS was unaffected in diaphragm interventions on KaplanMeier (57 vs. 81 months mean survival $p=0.135$ ). However, on cox regression, bilateral diaphragm resection significantly affected long-term survival in LAMNs $(p=0.037, \mathrm{RR}=2.230$, $95 \% \mathrm{CI}=1.052-4.730$, Figure 3).

\section{Discussion}

Diaphragmatic interventions are performed in nearly $50 \%$ of all CRS/IPC patients. With such a large cohort undergoing diaphragmatic CRS/IPC, factors that may pertain to a higher morbidity need to be identified. Many authors have examined the feasibility and safety of diaphragmatic interventions in CRS/IPC. However, there is a paucity of data available on the perioperative outcomes and long-term survival of bilateral diaphragm interventions.

In the literature, studies on diaphragmatic interventions are limited to ovarian pathology and attempts to compare the perioperative morbidity of diaphragm CRS/IPC to nondiaphragmatic CRS. Muallem and colleagues (10) found significant postoperative complications, infections and pleural effusions. Zapardiel and colleagues (14) attempted to quantify the effect of diaphragm resection and compared it to stripping in ovarian malignancies. In their postoperative univariate analysis, the resection group had more complete cytoreduction and significantly increased incidence of pleural effusions. Other authors such as Pounds and colleagues (15) performed a similar analysis.

The only other study that merits citation is by Franssen (9) and colleagues. They examined the short-term perioperative morbidity of diaphragmatic interventions in LAMNs. They discovered that intra-operative diaphragm interventions were associated with increased PRBC and 30-day mortality rate. Prolonged ICU-stay and length of hospital stay were also observed. Importantly, they are the only authors to mention bilateral diaphragm interventions. In their diaphragmatic cohort, 28 (31.4\%) of their 89 patients had bilateral diaphragm interventions. No subgroup analysis examining the effect of bilateral diaphragm interventions on perioperative mortality was performed.

After understanding the literature, our study attempted to identify whether bilateral diaphragm interventions are a risk factor for higher morbidity and mortality in CRS/IPC. Firstly, univariate analysis was conducted to quantify preoperative heterogeneity. This built the basis of our propensity matching method. Secondly, the matched data set was then utilized to measure intraoperative, postoperative and respiratory outcomes. Thirdly, an OS analysis based on tumor type was conducted. In essence, by comparing the long-term survival we were able to evaluate whether the short-term perioperative morbidity justifies the interventions.

The preoperative markers that determined heterogeneity between the diaphragm and non-diaphragm groups were carefully selected. These included ASA, sex, diagnosis, PSS, age and PCI. Categories such as intraoperative time and number of organs were deliberately not included because it was felt that they would be derivatives of the categories above. This would make the data of our study too specific and possibly not applicable to other populations.

In our analysis, significant heterogeneity was found in regard to diagnosis, PSS and PCI. LAMNs were more likely to undergo bilateral intervention, whereas colorectal cancers were unlikely to. This is a reflection of the strict PCI criterion of 10 or less for CRS + HIPEC in this diagnosis. Conversely, LAMNs patients had a significantly increased rate of bilateral intervention, reflecting the relaxed PCI bias in patient selection. Significantly increased numbers of patients with PSS 0 underwent bilateral intervention, whereas patients with PSS 1 were less likely to. This is likely the result of extensive dissection and preoperative PCI bias or technical difficulties in a redo versus a virgin abdomen or abdomen with limited resections. Unsurprisingly, the mean PCI was considerably higher in the bilateral diaphragmatic group. The data were propensity matched for diagnosis, PSS and PCI and yielded 165 matched pairs.

Univariate analysis was then conducted on these matched data. Our analysis identified significantly increased operative hours. Postoperatively, there were significantly increased incidences of RBC transfusion and grade III and IV complications. In terms of respiratory specific complications, a significantly increased incidence of pneumothorax was noted. Interestingly, OS was decreased in bilateral diaphragm interventions in LAMNs. We believe that the diaphragmatic 
disease in patients with LAMNs requiring bilateral diaphragm interventions, represents advanced infiltrative disease, which is contributing to the difference in OS.

Our study has several limitations. It is a retrospective, observational study with limitations inherent to this design. Firstly, unidentified confounding factors could influence results. Secondly, our data did not contain extubation times, which would be an important end-point to measure in terms of respiratory failure related to diaphragmatic instrumentation. Thirdly, our sample size may be too low to detect complications, which have low event rates. Lastly, this is a single institution study and the results may not be applicable to all institutions.

Despite the increase in short-term morbidity, bilateral diaphragm interventions allow for complete cyto-reduction and result in similar long-term survival to unilateral interventions.

\section{References}

1 Chua TC, Moran BJ, Sugarbaker PH, Levine EA, Glehen O, Gilly FN, Baratti D, Deraco M, Elias D, Sardi A, Liauw W, Yan TD, Barrios P, Gómez Portilla A, de Hingh IH, Ceelen WP, Pelz JO, Piso P, González-Moreno S, Van Der Speeten K and Morris DL: Early- and long-term outcome data of patients with pseudomyxoma peritonei from appendiceal origin treated by a strategy of cytoreductive surgery and hyperthermic intraperitoneal chemotherapy. J Clin Oncol 30: 2449-2456, 2012.

2 Yan TD, Deraco M, Baratti D, Kusamura S, Elias D, Glehen O, Gilly FN, Levine EA, Shen P, Mohamed F, Moran BJ, Morris DL, Chua TC, Piso P and Sugarbaker PH: Cytoreductive surgery and hyperthermic intraperitoneal chemotherapy for malignant peritoneal mesothelioma: multi-institutional experience. J Clin Oncol 27: 6237-6242, 2009.

3 Elit L, Oliver TK, Covens A, Kwon J, Fung MF, Hirte HW and Oza AM: Intraperitoneal chemotherapy in the first-line treatment of women with stage III epithelial ovarian cancer: a systematic review with metaanalyses. Cancer 109: 692-702, 2007.

4 Cashin PH, Mahteme H, Spång N, Syk I, Frödin JE, Torkzad M, Glimelius B and Graf W: Cytoreductive surgery and intraperitoneal chemotherapy versus systemic chemotherapy for colorectal peritoneal metastases: A randomised trial. Eur J Cancer 53: 155-162, 2016

5 Verwaal VJ, van Ruth S, de Bree E, van Sloothen GW, van Tinteren H, Boot $\mathrm{H}$ and Zoetmulder FA: Randomized trial of cytoreduction and hyperthermic intraperitoneal chemotherapy versus systemic chemotherapy and palliative surgery in patients with peritoneal carcinomatosis of colorectal cancer. J Clin Oncol 21: 3737-3743, 2003
6 Pelz Jo, Doerfer J, Decker M, Dimmler A, Hohenberger W and Meyer T: Hyperthermic intraperitoneal chemoperfusion (HIPEC) decrease wound strength of colonic anasatomosis in a rat model. Int J Colorectal Dis 22: 941-947, 2007.

7 Saxena A, Yan TD, Chua TC and Morris DL: Critical assessment of risk factors for complications after cytoreductive surgery and peroperative intraperitoneal chemotherapy for pseudomyxoma peritonei. Ann Surg Oncol 17: 1291-1301, 2010.

8 Chua TC, Saxena A, Schellekens JF, Liauw W, Yan TD, Fransi S, Zhao J and Morris DL: Morbidity and mortality outcomes of cytoreductive surgery and perioperative intraperitoneal chemotherapy at a single tertiary institution: towards a new perspective of this treatment. Ann Surg 251: 101-106, 2010.

9 Franssen B, Tabrizian P, Weinberg A, Romanoff A, Tuvin D, Labow D and Sarpel U: Outcome of cytoreductive surgery and hyperthermic intraperitoneal chemotherapy on patients with diaphragmatic involvement. Ann Surg Oncol 22: 1639-1644, 2015.

10 Muallem MZ, Almuheimid J, Richter R, Braicu EI, Osman S and Sehouli J: Diaphragmatic surgery in advanced ovarian, tubal and peritoneal cancer. A 7-Year retrospective analysis of the tumor bank ovarian cancer network. Anticancer Res 36: 4707-4713, 2016.

11 Sugarbaker PH: Surgical management of peritoneal carcinosis: diagnosis, prevention and treatment. Langenbecks Arch Chir 373: 189-196, 1988.

12 Jacquet $\mathrm{P}$ and Sugarbaker PH: Clinical research methodologies in diagnosis and staging of patients with peritoneal carcinomatosis. Cancer Treat Res 82: 359-374, 1996.

13 Dindo D. Demartines N and Clavein PA: Classification of surgical complications: a new proposal with evaluation in a cohort of 6336 patients and results of a survey. Ann Surg 240: 205-213, 2004.

14 Zapardiel I, Peiretti M, Zanagnolo V, Biffi R, Bocciolone L, Landoni F, Aletti G, Colombo N and Maggioni A: Diaphragmatic surgery during primary cytoreduction for advanced ovarian cancer: peritoneal stripping versus diaphragmatic resection. Int $\mathbf{J}$ Gynecol Cancer 21: 1698-703, 2011.

15 Pounds R, Phillips A, Kehoe S, Nevin J, Sundar S, Elattar A, Teo HG, Singh K and Balega J: Diaphragm disease in advanced ovarian cancer: Predictability of pre-operative imaging and safety of surgical intervention. Eur J Obstet Gynecol Reprod Biol 226: 47-53, 2018
Received August 20, 2018

Revised September 7, 2018 Accepted September 11, 2018 